\title{
Magnified single-balloon enteroscopy in the diagnosis of intestinal follicular lymphoma: a case series
}

\author{
Kenichiro Takahashi ${ }^{1}$, Shigeki Bamba ${ }^{2}$, Masahiro Kawahara ${ }^{1}$, Atsushi Nishida ${ }^{1}$, Osamu Inatomi ${ }^{1}$, \\ Masaya Sasaki ${ }^{2}$, Tomoyuki Tsujikawa ${ }^{3}$, Ryoji Kushima ${ }^{4}$, Mitsushige Sugimoto ${ }^{5}$, Katsuyuki Kitoh ${ }^{1}$, \\ Akira Andoh ${ }^{1}$ \\ ${ }^{1}$ Department of Medicine, ${ }^{2}$ Division of Clinical Nutrition, ${ }^{3}$ Department of Comprehensive Internal Medicine and ${ }^{4}$ Division of Diagnostic \\ Pathology, and ${ }^{5}$ Division of Digestive Endoscopy, Shiga University of Medical Science, Otsu, Japan
}

The objective of this study was to evaluate the magnified endoscopic findings in the diagnosis of follicular lymphoma in the small intestine in comparison with those of intestinal follicular lymphoma and lymphangiectasia. Four patients with follicular lymphoma and 3 with lymphangiectasia in the small intestine were retrospectively analyzed. A prototype magnifying singleballoon enteroscope was used. The findings of the intestinal follicular lymphoma and lymphangiectasia were retrospectively analyzed to determine the magnified endoscopic findings of follicular lymphoma in the small intestine. Opaque white granules were observed in 3 of the 4 patients with follicular lymphoma. Magnified narrow-band imaging (NBI) of the opaque white granules showed stretched microvessels, which had a diminutive tree-like appearance. The remaining patient had no opaque white granules and only displayed whitish villi. Magnified NBI observation of the whitish villi revealed the absence of marginal villus epithelium, which was confirmed by histology. The magnified NBI enteroscopy revealed the diminutive tree-like appearance on the opaque white granules and the absence of marginal villus epithelium of the whitish villi in intestinal follicular lymphoma. These findings may be useful in diagnosing follicular lymphoma. (Intest Res 2018;16:628-634)

Key Words: Balloon-assisted enteroscopy; Narrow band imaging; Intestine, small

\section{INTRODUCTION}

The use of a magnifying endoscopy to evaluate gastrointestinal diseases of the digestive tract has many advantages, particularly for those in the esophagus, stomach and large intestine. The use of magnifying esophagogastroduodenoscopes and colonoscopes is now widespread, and they are considered indispensable tools for the diagnosis of gastrointestinal lesions, especially in the diagnosis of neoplastic lesions. Although the ability to access the small bowel has

Received January 11, 2018. Revised April 30, 2018.

Accepted April 30, 2018.

Correspondence to Shigeki Bamba, Division of Clinical Nutrition, Shiga

University of Medical Science, Seta-Tsukinowa, Otsu 520-2192, Japan. Tel:

+81-77-548-2217, Fax: +81-77-548-2219, E-mail: sb@belle.shiga-med.

ac.jp dramatically improved since the advent of double-balloon ${ }^{1}$ and single-balloon ${ }^{2}$ enteroscopes, the advantages of the magnifying enteroscope in the evaluation of diseases of the small intestine have not been clarified.

With the development of balloon-assisted enteroscopy or video capsule endoscopy, gastrointestinal follicular lymphomas are now more frequently found in the small intestine, especially in the proximal small intestine. ${ }^{3}$ Typical findings of follicular lymphoma on conventional observation using balloon-assisted enteroscopy include opaque white granules and whitish villi. ${ }^{4,5}$ Although duodenal follicular lymphomas have been evaluated by magnified endoscopy, ${ }^{6,7}$ none of these reports has shown the usefulness of magnified endoscopy in the diagnosis of follicular lymphoma. ${ }^{8}$ Further, no report has described the use of magnified endoscopy to

๑ Copyright 2018. Korean Association for the Study of Intestinal Diseases. All rights reserved.

This is an Open Access article distributed under the terms of the Creative Commons Attribution Non-Commercial License (http://creativecommons.org/licenses/by-nc/4.0)

which permits unrestricted non-commercial use, distribution, and reproduction in any medium, provided the original work is properly cited. 
observe follicular lymphoma in other parts of the small intestine excluding of duodenum. It is important to distinguish follicular lymphoma from lymphangiectasia, a lesion which also exhibits whitish villi, and it is unknown whether these diseases in the small intestine can be distinguished on magnifying endoscopy.

Magnified observation of the small intestine was first reported in 1980 by Tada et al. ${ }^{9}$ However, the scope (SIF-M; Olympus, Tokyo, Japan) required insertion using the ropeway method. Recently, we were given the opportunity to use a prototype of the magnified single-balloon enteroscope (SIFY0007; Olympus), which enables detailed morphological observation of the villi and microvessels. ${ }^{10}$ Here, we report a case series of patients with intestinal follicular lymphoma and lymphangiectasia who underwent magnifying endoscopy to determine the specific findings of follicular lymphoma of the small intestine.

\section{CASE REPORT}

We report 4 cases of follicular lymphoma and 3 cases of lymphangiectasia who were examined between September 2013 and April 2017 using a magnifying single-balloon enteroscope (SIF-Y0007). SIF-Y0007 provides a magnification of up to $80 \times$ and other specifications of SIF-Y0007 and SIFQ260 are shown in Supplementary Table 1. Although only
$0.7 \mathrm{~mm}$ wider than the SIF-Q260 in outer diameter, the SIFY0007 is also characterized by passive bending and high force transmission. The SIF-Y0007, therefore, allows a similar degree of deep insertion as the SIF-Q260 and can be used in the routine examination. The study was approved by the Ethics Committee of the Shiga University of Medical Science (approval number: 29-116). The Ethics Committee of the Shiga University of Medial Science waived the need for consent from individuals. The magnifying single-balloon enteroscopy was performed by 2 specialists (S.B. and K.T.).

Abdominal CT was performed to examine elevated CA199 (case 1), body weight loss (case 2), and abdominal pain (cases 3 and 4). Abdominal lymphadenopathy was revealed by abdominal CT in all patients. The small bowel examination was performed because there were no significant findings by esophagoduodenoscopy and colonoscopy. The route of insertion was decided by the findings of capsule endoscopy and CT. In cases 1 and 3, magnifying single-balloon enteroscopy was performed with both anterograde and retrograde approaches. In cases 2 and 4, only anterograde insertion was performed.

The biopsy specimens of all cases were evaluated by histopathology. Immunohistochemical staining for CD10, CD20, bcl-2, bcl-6, CD3, CD5 and cyclin D1 was performed. As a comparison, we also present 3 cases with whitish villi that were diagnosed as lymphangiectasia by histopathological

Table 1. Summary of Cases

\begin{tabular}{|c|c|c|c|c|c|c|c|}
\hline \multirow{2}{*}{ Characteristics } & \multicolumn{3}{|c|}{ Follicular lymphoma } & \multirow[b]{2}{*}{ Case 4} & \multicolumn{3}{|c|}{ Lymphangiectasia } \\
\hline & Case 1 & Case 2 & Case 3 & & Case 5 & Case 6 & Case 7 \\
\hline Corresponding figures & Fig. 1 & Fig. $2 A, B$ & Fig. 2C, D & Fig. 3 & Fig. 4 & Fig. 5A, B & Fig. 5C, D \\
\hline Age (yr) & 54 & 58 & 73 & 63 & 58 & 82 & 15 \\
\hline Sex & Male & Female & Male & Male & Male & Female & Male \\
\hline Location & $J / l$ & J & $J / l$ & J & 1 & J & $J$ \\
\hline Soluble interleukin-2 receptor (U/mL) & 262 & 2,610 & 10,800 & 283 & - & - & - \\
\hline No. of affected areas & 5 & 1 & 5 & 2 & 1 & 1 & Diffuse \\
\hline Whitish villus & Yes & Yes & Yes & Yes & Yes & Yes & Yes \\
\hline $\begin{array}{l}\text { Absence of marginal villus epithelium } \\
\text { (magnified NBI observation) }\end{array}$ & No & No & No & Yes & No & No & No \\
\hline Opaque white granules & Yes & Yes & Yes & No & No & No & No \\
\hline $\begin{array}{l}\text { Diminutive tree-like appearance } \\
\text { (magnified NBI observation) }\end{array}$ & Yes & Yes & Yes & No & No & No & No \\
\hline Enlarged villi & Yes & Yes & Yes & No & No & Yes & No \\
\hline $\begin{array}{l}\text { Coiled or gyrus-like microvessels } \\
\text { (magnified NBI observation) }\end{array}$ & Yes & No & Yes & No & No & Yes & No \\
\hline Follicular lymphoma grade & $\mathrm{G} 1$ & G1 & G1 & $\mathrm{G} 2$ & - & - & - \\
\hline
\end{tabular}

J, jejunum; I, ileum; NBI, narrow-band imaging. 

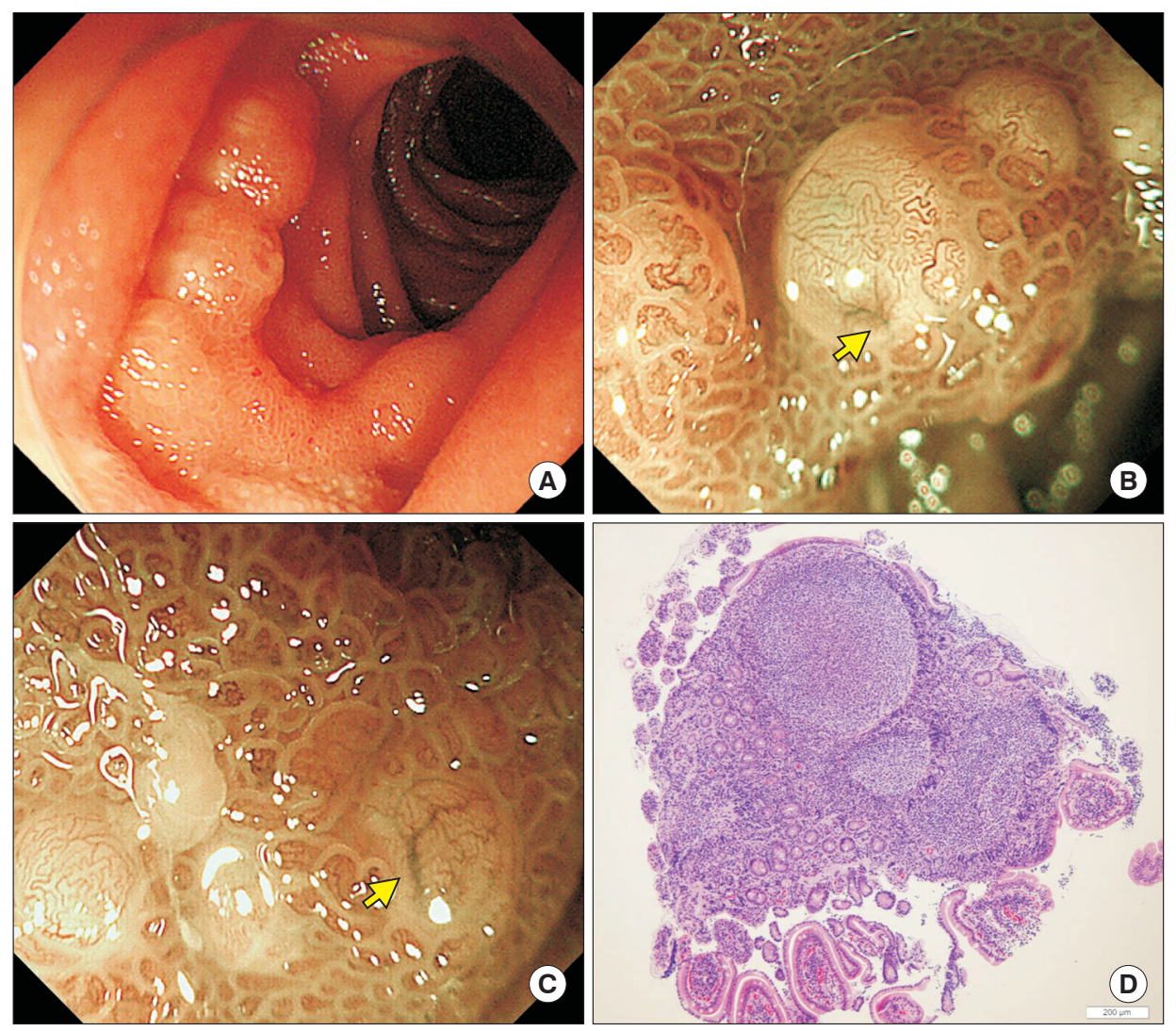

Fig. 1. Case 1: follicular lymphoma of the jejunum. (A) White light observation of the jejunal follicular lymphoma. $(B, C)$ Narrowband imaging with magnified endoscopy revealed confluent hemispherical elevation without marginal villus epithelium. Diminutive tree-like appearance of microvessels (yellow arrow) and gyrus-like microvessels were observed. (D) Histological images of biopsy samples taken from the opaque white granules. Lymphoid follicles were present in the lamina propria and caused stretching of the epithelium (x40).

examination of a biopsy specimen. On immunohistochemical staining, cases of follicular lymphoma were positive for CD10, CD20, bcl-2 and bcl-6, and negative for CD3, CD5 and cyclin D1, while cases of lymphangiectasia were negative for these markers.

Because follicular lymphoma and lymphangiectasia both show whitish villi, it is important to distinguish between them. Jejunal lesions were present in all cases of follicular lymphoma (Table 1, Figs. 1-3). Three of the 4 cases of follicular lymphoma had multiple lesions in the jejunum and ileum. Opaque white granules were found on conventional white light observation in cases 1, 2, and 3 (Figs. 1A, 2A, and C). Opaque white granules reflect the tumorous growth of lymphoid follicles in the lamina propria (Fig. 1D). The epithelium was stretched on the surface of the lymphoid follicle because of the tumorous growth of lymphoid follicles (Fig. 1D).

In case 4, we only found whitish villi but not opaque white granules (Fig. 3A-C). Case 4 was diagnosed as follicular lymphoma by histological evaluation. However, it was difficult to distinguish follicular lymphoma from lymphangiectasia without magnifying endoscopic observation. Magnified endoscopic observation revealed the absence of marginal villus epithelium (Fig. 3C). This finding was also confirmed by histology (Fig. 3D).

Lymphangiectasia is characterized by patchy and dense lesions without a clear margin (case 5, Fig. 4), slight submucosal tumor-like elevation (case 6, Fig. 5A and B) and scattered white spots (case 7, Fig. 5C and D). As shown in cases 5,6 , and 7 , marginal villus epithelium was observed on the whitish villi of lymphangiectasia.

Vascular architecture was clearly depicted on magnified narrow-band imaging (NBI) observation. On magnified NBI observation, a diminutive tree-like appearance of microvessels was observed on the surface of the opaque white granules (Figs. 1B and C, 2B and D). On the other hand, coiled or gyrus-like microvessels were observed in both follicular lymphoma and lymphangiectasia (Figs. 1B and C, 5B).

\section{DISCUSSION}

The advantages of this study include the novel application of magnifying single-balloon enteroscope to follicular lymphoma in the small intestine to differentiate them from lymphangiectasia. In addition to the presence of opaque white granules on conventional images, magnified NBI observation revealed the diminutive tree-like appearance on the opaque white granules and the absence of marginal villus epithelium 

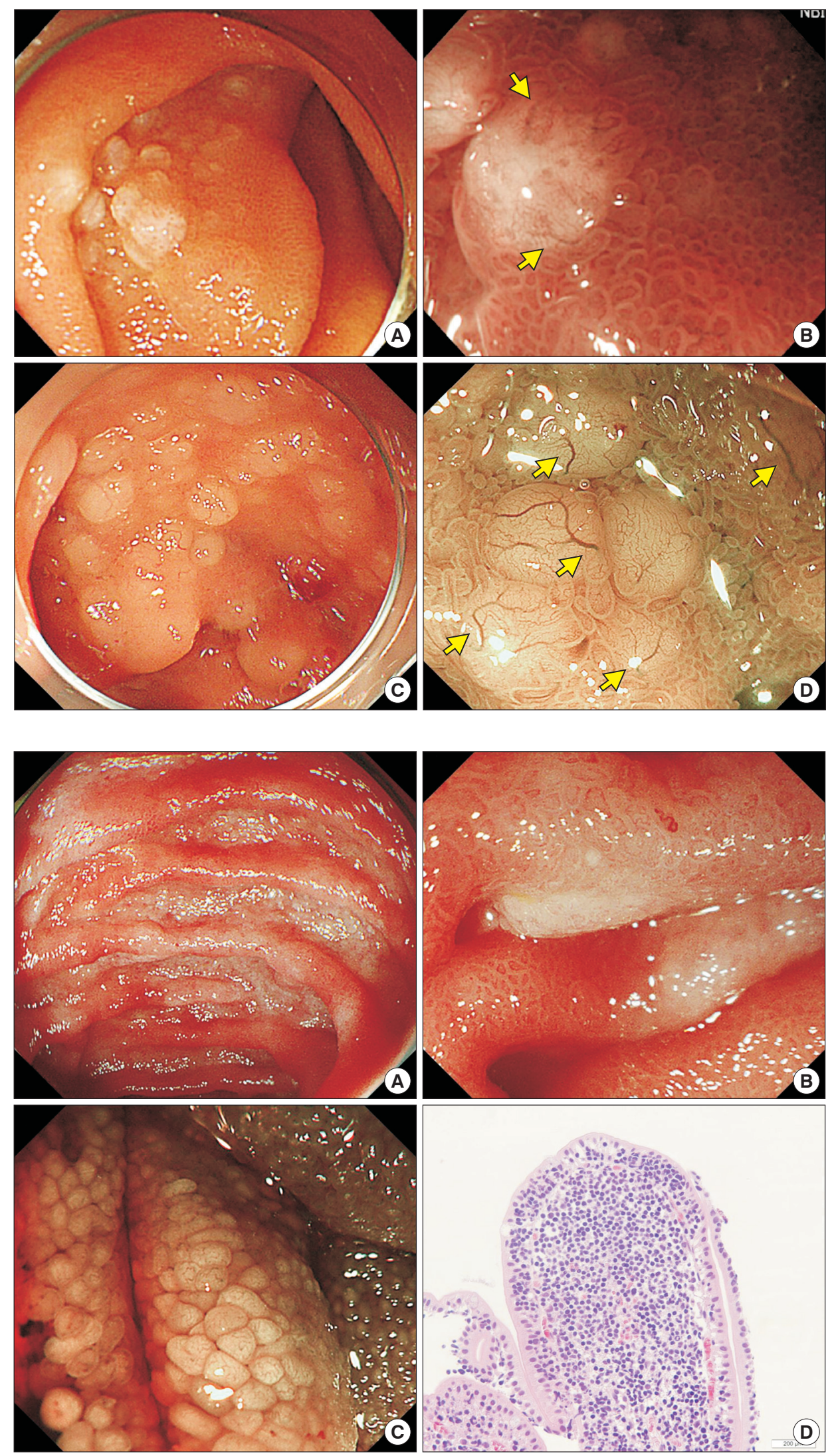

Fig. 2. Case $2(A, B)$ and case $3(C, D)$ : follicular lymphoma of the jejunum. (A) White light observation of the jejunal follicular Iymphoma. (B) Narrow-band imaging magnified endoscopy revealed confluent hemispherical elevation. Diminutive tree-like appearance (yellow arrows) was observed. (C) White light image of the jejunal follicular lymphoma. (D) Narrow-band imaging magnified observation revealed diminutive tree-like appearance (yellow arrows) on the opaque white granules.

Fig. 3. Case 4: follicular lymphoma of the jejunum. (A) Jejunal lesion of follicular lymphoma seen on white light observation. (B) Magnified observation under white light demonstrated relatively homogenous whitish villi. (C) Narrow-band imaging magnified endoscopy demonstrated whitish villi without marginal villus epithelium. (D) Histological images of biopsy samples taken from whitish villi without marginal villus epithelium. Lymphoma cells infiltrated the lamina propria, and the epithelial cells were replaced by lymphoma cells at the tip of villi (x200). 

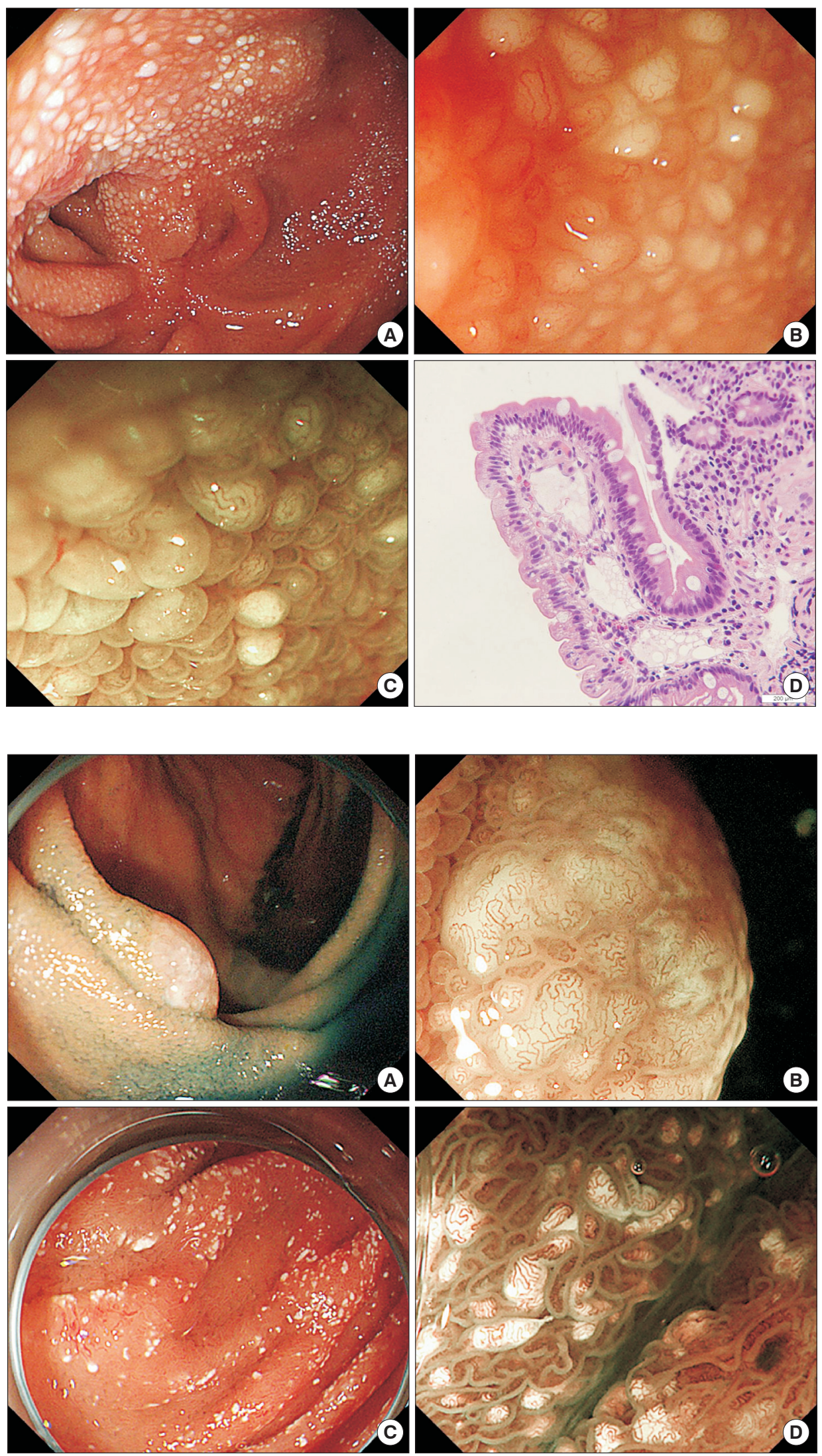

Fig. 4. Case 5: Iymphangiectasia. (A) A patient with $\mathrm{CD}$ underwent single-balloon enteroscopy for endoscopic balloon dilatation for intestinal stricture. White light view of the ileum demonstrated a scar lesion and whitish villi in the ileum. $(B, C)$ White light and narrow-band imaging magnified endoscopy revealed whitish villi with marginal villus epithelium. (D) Histological images of biopsy samples taken from the whitish villi. Markedly dilated lymphatics were present in the lamina propria ( $\times 200)$.

Fig. 5. Case $6(A, B)$ and case $7(C, D)$ : lymphangiectasia. (A) The patient underwent single-balloon enteroscopy to identify the source of gastrointestinal bleeding. Lymphangioma was observed as a smooth elevated lesion with whitish villi. (B) Narrow-band imaging magnified endoscopy revealed enlarged whitish villi with gyruslike microvessels. (C) A patient who had undergone the Fontan procedure for single ventricle of right ventricular morphology, pulmonary artery occlusion and patent ductus arteriosus developed protein-losing enteropathy, and single-balloon enteroscopy was therefore performed. Scattered white spots were observed in the jejunum. (D) Narrow-band imaging magnified observation revealed scattered white spots within the tips of villi. 
of the whitish villi, which were observed in only cases of follicular lymphoma. The disadvantages of this study are the small number of patients and the prototype nature of the magnifying enteroscope which is not commercially available.

In describing endoscopic findings of lymphangiectasia, scattered white spots were firstly reported by Asakura et al. ${ }^{11}$ indicating jejunal lymphangiectasia using conventional enteroscope from distant view observation. The "spots" of scattered white spots means individual whitish villus scattered in the intestine. On the other hand, follicular lymphoma forms white hemispherical elevation composed of lymphoma cells, which we describe as "opaque white granules." As for the previous reports, Norimura et al. ${ }^{7}$ describe the white hemispherical elevation as "opaque white spots." However, the "spots" of opaque white spots does not mean villi as Asakura et al. described in scattered white spots. Besides, Iwamuro et al. ${ }^{5,12}$ described the white hemispherical elevation as "white nodules" or "whitish granules." Therefore, we describe white hemispherical elevation as "opaque white granules" in this study.

On white light observation, opaque white granules were observed in 3 of 4 cases ( $75 \%$ ) of follicular lymphoma. Opaque white granules showed relatively coarse granularity in comparison with lymphangiectasia which displayed whitish fine granularity. Follicular lymphoma in the small intestine forms a main lesion in the lamina propria of the mucosa and the lymphoid follicles form a hemispherical elevation with increasing tumor size. Magnified NBI endoscopic observation of opaque white granules allowed visualization of the abnormal microvessels, which are described as having a treelike appearance. The tree-like appearance was first reported as a feature in the detection of gastric mucosa-associated lymphoid tissue lymphoma. Nonaka et al. ${ }^{13}$ described the tree-like appearance of abnormal blood vessels, resembling branches extending from the trunk of a tree on shiny mucosa in which glandular structure was lost. In our cases of follicular lymphoma in the small intestine, we found diminutive tree-like appearance in 3 of 4 cases (75\%) as a tapered and branched relatively thick blood microvessel extending from the base of the opaque white granules towards the top. Enlarged lymphoid follicles push up and stretch the microvessels to the surface layer and recognized as diminutive treelike appearance.

Previous reports of follicular lymphoma, including cases in the duodenum, described the vascular findings of follicular lymphoma as: enlarged villi; a coiled, elongated vascular pattern within the villi; and dilatation of microvessels. ${ }^{4,7,8,14}$
However, these findings were also observed in our cases of lymphangiectasia. Diminutive tree-like appearance on the opaque white granules may be the important vascular finding of follicular lymphoma in the small intestine.

In previous reports and in our present case series also, many cases of the follicular lymphoma had opaque white granules. However, other cases showed whitish villi only, and no opaque white granules. ${ }^{8,14}$ In the cases of lymphangiectasia, the white color of the villous center reflects the dilatation of the central lacteal. In contrast, follicular lymphoma in which only whitish villi are observed is thought to predominantly represent lepidic tumor growth. In this case, the whitish villi are a result of tumor cells that fill the villi. In addition, the marginal villus epithelium disappears because epithelial cells are replaced by lymphoma cells at the tip of the villi. Identifying the presence of the marginal villus epithelium is important when whitish villi are present. Therefore, the absence of marginal villus epithelium is less frequent $(25 \%$ in our case series) but may be important findings.

In conclusion, the magnified NBI enteroscopy revealed the diminutive tree-like appearance on the opaque white granules and the absence of marginal villus epithelium of the whitish villi in intestinal follicular lymphoma. These findings may be useful in diagnosing follicular lymphoma of the small intestine. However, the final diagnosis of small intestinal lymphoma is a pathologic evaluation of biopsy specimen. Therefore, a more aggressive biopsy should be considered if one encounters these findings.

\section{FINANCIAL SUPPORT}

The authors received no financial support for the research, authorship, and/or publication of this article.

\section{CONFLICT OF INTEREST}

The magnifying single-balloon enteroscope (SIF-Y0007) was loaned free of charge by the Olympus Corporation.

\section{AUTHOR CONTRIBUTION}

Conceptualization: Kenichiro Takahashi. Methodology: Kenichiro Takahashi, Shigeki Bamba. Formal analysis: Shigeki Bamba. Investigation: all authors. Writing - original draft: Kenichiro Takahashi. Writing - review and editing: Shigeki Bamba. Supervision: Akira Andoh. Approval of final manuscript: all authors. 


\section{REFERENCES}

1. Yamamoto H, Sekine Y, Sato Y, et al. Total enteroscopy with a nonsurgical steerable double-balloon method. Gastrointest Endosc 2001;53:216-220.

2. Tsujikawa T, Saitoh Y, Andoh A, et al. Novel single-balloon enteroscopy for diagnosis and treatment of the small intestine: preliminary experiences. Endoscopy 2008;40:11-15.

3. Yamamoto S, Nakase H, Yamashita K, et al. Gastrointestinal follicular lymphoma: review of the literature. J Gastroenterol 2010;45:370-388.

4. Chowdhury M, Endo M, Chiba T, et al. Characterization of follicular lymphoma in the small intestine using double-balloon endoscopy. Gastroenterol Res Pract 2009;2009:835258.

5. Iwamuro M, Okada H, Kawano S, et al. A multicenter survey of enteroscopy for the diagnosis of intestinal follicular lymphoma. Oncol Lett 2015;10:131-136.

6. Inoue N, Isomoto H, Shikuwa S, Mizuta Y, Hayashi T, Kohno S. Magnifying endoscopic observation of primary follicular lymphoma of the duodenum by using the narrow-band imaging system. Gastrointest Endosc 2009;69:158-159.

7. Norimura D, Isomoto H, Imaizumi Y, et al. Case series of duodenal follicular lymphoma, observed by magnified endoscopy with narrow-band imaging. Gastrointest Endosc 2011;74:428434.
8. Iwamuro M, Okada H, Takata K, et al. Magnified endoscopic features of duodenal follicular lymphoma and other whitish lesions. Acta Med Okayama 2015;69:37-44.

9. Tada M, Suyama Y, Shimizu T, et al. Observation of the villi with the magnifying entero-colonoscopes. Gastroenterol Endosc 1980;22:647-654.

10. Murata M, Bamba S, Takahashi K, et al. Application of novel magnified single balloon enteroscopy for a patient with Cronkhite-Canada syndrome. World J Gastroenterol 2017;23:41214126.

11. Asakura H, Miura S, Morishita T, et al. Endoscopic and histopathological study on primary and secondary intestinal lymphangiectasia. Dig Dis Sci 1981;26:312-320.

12. Iwamuro M, Okada H, Takata K, et al. Magnifying endoscopic observation of duodenal involvement of follicular lymphoma before and after chemotherapy. Intern Med 2015;54:1741-1745.

13. Nonaka K, Ishikawa K, Arai S, et al. A case of gastric mucosaassociated lymphoid tissue lymphoma in which magnified endoscopy with narrow band imaging was useful in the diagnosis. World J Gastrointest Endosc 2012;4:151-156.

14. Iwamuro M, Okuda M, Yumoto E, et al. Magnifying endoscopy for intestinal follicular lymphoma is helpful for prompt diagnosis. Gut Liver 2013;7:258-261. 